\title{
Model-independent diagnostics of highly reddened Milky Way star clusters: age calibration ${ }^{\star}$ (Research Note)
}

\author{
Y. Beletsky ${ }^{1}$, G. Carraro ${ }^{1,2}$, and V. D. Ivanov ${ }^{1}$ \\ 1 ESO, Alonso de Cordova 3107, Santiago de Chile, Chile \\ e-mail: [ybialets; gcarraro; vivanov]@eso.org \\ 2 Dipartimento di Astronomia, Università di Padova, Vicolo Osservatorio 2, 35122 Padova, Italy
}

Received 15 June 2009 / Accepted 11 August 2009

ABSTRACT

\begin{abstract}
Context. The next generation near- and mid-infrared Galactic surveys will yield a large number of new highly obscured star clusters. Detailed characterization of these new objects with spectroscopy is time-consuming.

Aims. Diagnostic tools that will be able to characterize clusters based only on the available photometry will be needed to study large samples of the newly found objects.

Methods. The brightness difference between the red clump and the main-sequence turn-off point have been used as a modelindependent age calibrator for clusters with ages from a few $10^{8}$ to $10^{10} \mathrm{yr}$ in the optical. Here we apply for the first time the method in the near-infrared.

Results. We calibrated this difference in $K$-band, which is likely to be available for obscured clusters, and we apply it to a number of test clusters with photometry comparable to the one that will be yielded by the current or near-future surveys.

Conclusions. The new calibration yields reliable ages over the range of ages for which the red clump is present in clusters. The slope of the relation is smoother than that of the corresponding $V$-band relation, reducing the uncertainty in the age determinations with respect to the optical ones.
\end{abstract}

Key words. open clusters and associations: general - surveys

\section{Introduction}

The recent advance in the infrared (IR) instrumentation made it possible to carry out large scale sky surveys. The Two Micron All Sky Survey (2MASS; Skrutskie et al. 2006) was the first one to provide a deep look into the most obscured regions of the Milky Way. A number of new clusters were found in 2MASS (i.e. Dutra \& Bica 2000; Ivanov et al. 2002; Bica et al. 2003; Borissova et al. 2003). Currently, the UKIRT Infrared Deep Sky Survey (UKIDSS; Lawrence et al. 2007) is creating a map of selected Northern sky regions, typically $\sim 5$ mag deeper than the 2MASS, and in the near future the Visible and Infrared Survey Telescope for Astronomy (VISTA; Emerson et al. 2006; Dalton et al. 2006) will provide similar coverage in the South. These projects include specialized multicolor Galactic plane surveys: UKIDSS Galactic plane surveys (GPS; Lucas et al. 2008) and VISTA variables in the Via Lactea (VVV; Minniti et al. 2006) which are expected to reveal a large number of new star clusters.

Their characterization is a time-consuming process, requiring spectroscopy in the near-IR, because of the strong intervening dust absorption $\left(A_{K} \sim 0.1 \times A_{V}\right.$; Rieke \& Lebofsky $1985)$. This prompted us to attempt to develop various methods to determine the cluster parameters from the photometry alone. For example, the metal abundances of globular clusters is easily measured with an error of $\sigma([\mathrm{Fe} / \mathrm{H}]) \sim 0.2$ dex (i.e.

* Original data are only available in electronic form at the CDS via anonymous ftp to cdsarc.u-strasbg.fr (130.79.128.5) or via http://cdsweb.u-strasbg.fr/cgi-bin/qcat?]/A+A/508/1279
Ferraro et al. 1999; Ivanov \& Borissova 2002) from the slope of the RGB on the color-magnitude diagrams (CMD).

In this Note we focus on a new age calibrator based on the $K$-band. difference between the clump and the turn off point (TO) in the CMD of star clusters older than about $300 \mathrm{Myr}$, which will be widely used to get a quick and approximate age values for newly discovered IR star clusters.

The layout of the paper is as follows. In Sect. 2 we introduce the concept of age calibrator as derived and used in the optical regime. Section 3 describes the sample of very well know star clusters on which we base our new age calibrator. A few analytical relations are given in Sect. 4. These relations are then applied to a few test-cases in Sect. 5. Section 6, finally, summarizes out findings.

\section{Age calibrations for open star clusters}

The magnitude difference $\Delta V$ between the main sequence (MS) TO and the clump of He burning stars is a well known age indicator for Galactic open clusters. The very requirement to have a well-defined red clump limits it to clusters older than $\sim 300 \mathrm{Myr}$ when the He burning stars appear. However, the method is distance and reddening independent, as demonstrated early on by Cannon (1970), and it is not surprising that it has been widely used ever since (see, for instance, Carraro \& Chiosi 1994; Salaris et al. 2004).

Different, more sophisticated versions of this calibrator have been devised in the past, in the optical regime, by Anthony-Twarog \& Twarog (1985) and Phelps et al. (1994). 
Table 1. Parameters of the clusters' sample.

\begin{tabular}{lcccccr}
\hline \hline \multicolumn{1}{c}{ Name } & $\begin{array}{c}\text { RA } \\
\text { hh:mm:ss }\end{array}$ & $\begin{array}{c}\text { Dec } \\
:^{\prime}:{ }^{\prime \prime}\end{array}$ & $\begin{array}{c}\text { Age } \\
{[\mathrm{Gyr}]}\end{array}$ & {$[\mathrm{Fe} / \mathrm{H}]$} & $\begin{array}{c}\Delta K \\
{[\mathrm{mag}]}\end{array}$ & Ref. \\
\hline NGC 188 & $00: 47: 28$ & +22.384 & 6.0 & $+0.01 \pm 0.08$ & $4.0 \pm 0.15$ & 1 \\
NGC 752 & $01: 57: 41$ & -23.254 & 1.6 & $-0.16 \pm 0.10$ & $2.7 \pm 0.20$ & 2 \\
NGC 2420 & $07: 38: 23$ & +19.634 & 2.1 & $-0.26 \pm 0.07$ & $3.3 \pm 0.20$ & 3 \\
NGC 2477 & $07: 52: 10$ & -05.850 & 0.8 & $+0.07 \pm 0.03$ & $1.9 \pm 0.25$ & 4 \\
NGC 2506 & $08: 00: 01$ & +09.935 & 1.9 & $-0.37 \pm 0.02$ & $3.1 \pm 0.25$ & 5 \\
NGC 2509 & $08: 00: 48$ & +05.820 & 1.2 & $+0.00 \pm 0.10$ & $2.5 \pm 0.20$ & 6 \\
M 67 & $08: 51: 18$ & +31.896 & 4.0 & $+0.00 \pm 0.01$ & $3.8 \pm 0.10$ & 7 \\
NGC 3680 & $11: 25: 38$ & +16.918 & 1.8 & $-0.16 \pm 0.03$ & $2.9 \pm 0.15$ & 8 \\
NGC 6134 & $16: 27: 46$ & -00.200 & 1.0 & $+0.18 \pm 0.10$ & $2.0 \pm 0.15$ & 9 \\
NGC 6253 & $16: 59: 05$ & -06.260 & 3.0 & $+0.36 \pm 0.07$ & $3.7 \pm 0.15$ & 10 \\
IC 4651 & $17: 24: 49$ & -07.907 & 1.1 & $-0.11 \pm 0.01$ & $2.1 \pm 0.20$ & 11 \\
NGC 6819 & $19: 41: 18$ & +08.481 & 2.5 & $+0.09 \pm 0.03$ & $3.4 \pm 0.25$ & 12 \\
NGC 6939 & $20: 31: 30$ & +12.304 & 2.2 & $+0.02 \pm 0.05$ & $3.2 \pm 0.15$ & 13 \\
NGC 7789 & $23: 57: 24$ & -05.385 & 1.4 & $-0.10 \pm 0.05$ & $2.4 \pm 0.20$ & 14 \\
\hline
\end{tabular}

The last column gives a reference to the literature source of the adopted cluster age and metallicity.

1) Fornal et al. (2007); 2) Anthony-Twarog \& Twarog (2006); 3) Anthony-Twarog et al. (2006); 4) Kassis et al. (1997); 5) Marconi et al. (1997); 6) Carraro \& Costa (2007); 7) Sarajedini et al. (2009); 8) Anthony-Twarog \& Twarog (2004); 9) Bruntt et al. (1999); 10) Twarog et al. (2003); 11) Meibon et al. (2002); 12) Kalirai et al. (2001); 13) Andreuzzi et al. (2004); 14) Vallenari et al. (2000).

They also include the color difference between the TO and the red giant branch (RGB). However, their application is more limited because usually the RGBs of open clusters are poorly populated, and the RGB colors are ill-defined.

The simple difference $\Delta V$ between the red clump and TO - the two major features in the CMD - has the advantage of providing a quick age estimate even from un-calibrated CMDs (Phelps et al. 1994; Salaris et al. 2004). As described by Carraro \& Chiosi (1994) this indicator shows a slight metallicity dependence in the optical: $\Delta V$ at fixed age is larger for higher metallicity clusters than for lower metallicity ones.

The aim of the present work is to extend this calibrator to the near-IR, where the metallicity effect should be weaker. We will use the 2MASS $K_{\mathrm{S}}$ filter as the least affected by the extinction, and throughout the Note will mark it as $K$, for simplicity.

\section{Sample selection and indicator definition}

We selected 14 open clusters with 2MASS photometry, with reliable age and metallicity estimates, spanning a wide range of the parametric space (Table 1). The data come from the latest edition of the WEBDA ${ }^{1}$ database maintained by E. Paunzen at Vienna University. Throughout the paper we adopted a conservative estimates of the uncertainty in the age of $15 \%$ and a factor of 2 for the abundance $[\mathrm{Fe} / \mathrm{H}]$.

Some of the clusters suffer from significant fore- and background contamination since they are located low onto the Galactic plane. To minimize it, we built up homogeneous CMDs considering only the stars within the accepted cluster radii estimates from Dias et al. (2002). The CMDs for M67 and NGC 2506 - two clusters with rather different ages and abundances - are shown in Fig. 1.

Next, we identified the clump and TO magnitudes, respectively at $K^{\text {clump }}=8.00 \pm 0.05$, and $K^{\mathrm{TO}}=11.80 \pm 0.10 \mathrm{mag}$, for M 67. This implies $\Delta K=3.8 \pm 0.1 \mathrm{mag}$. For NGC 2506 we found $K^{\text {clump }}=10.85 \pm 0.15$, and $K^{\mathrm{TO}}=14.00 \pm 0.20 \mathrm{mag}$, yielding

\footnotetext{
${ }^{1}$ http://www. univie.ac.at/webda/navigation.html
}

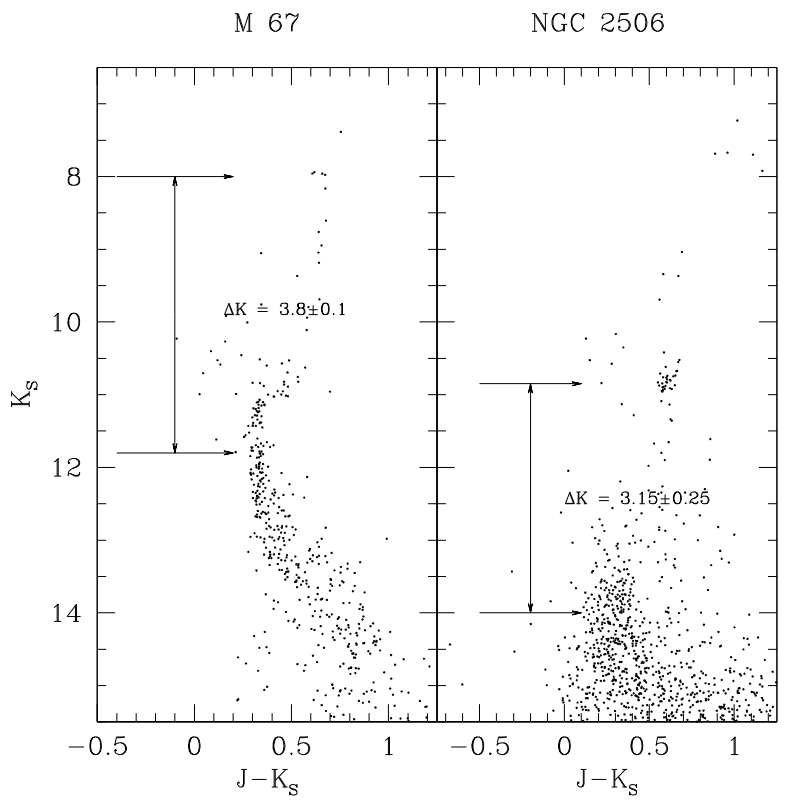

Fig. 1. Definition of the parameter $\Delta K$. The left panel shows the CMD of M 67 (solar metallicity, 4 Gyr old), the right shows the CMD of NGC 2506 (significantly subsolar metallicity, twice younger than M 67).

$\Delta K=3.15 \pm 0.25 \mathrm{mag}$. These examples illustrate that for an old cluster like M67 the definition of the clump magnitude is easier despite the smaller number of He burning stars, while the clump at younger ages is wider both in color and magnitude due to evolutionary effects (Girardi et al. 2000).

The measured values of $\Delta K$ are also listed in Table 1 . Their uncertainties were obtained tentatively, by eye. These take into account the broadening of features in the CMD due to photometric errors, patchy extinction and unresolved binaries. The experience shows that such estimates can be even more reliable than the automated ones (i.e. see the discussion in Sect. 4 of Phelps \& Janes 1994). The age (logarithm) versus $\Delta K$ relation is plotted in Fig. 2.

We emphasize that this IR relation looks smoother than the optical counterpart (Carraro \& Chiosi 1994) mainly because of the significantly weaker metallicity dependence (see below). Furthermore, the smooth points distribution in Fig. 2 makes any analytical fit through the data easier and more robust.

\section{Calibration}

An analytical representation of the age versus $\Delta K$ relation is needed to facilitate the derivation of cluster ages. The relation appears non-linear, prompting us to fit a higher order polynomial:

$\log ($ Age $)=9.09(0.29)+0.24(0.20) \times \Delta K+0.10(0.03) \times(\Delta K)^{2}(1)$

with rms $=0.28$. The reduced $\chi^{2}$ of the fit is 0.08 .

For comparison, the linear fit to the data is:

$\log ($ Age $)=8.27(0.1)+0.35(0.03) \times \Delta K$

with rms $=0.36$. Numbers in brackets are corresponding uncertainties. The reduced $\chi^{2}$ of this fit is 0.04 .

The age in these relations is expressed in Gyr. The two fits are nearly indistinguishable for $2.5 \leq \Delta K \leq 3.5 \mathrm{mag}$ - the deviations from the linearity occur in fact at the extremes of the age 


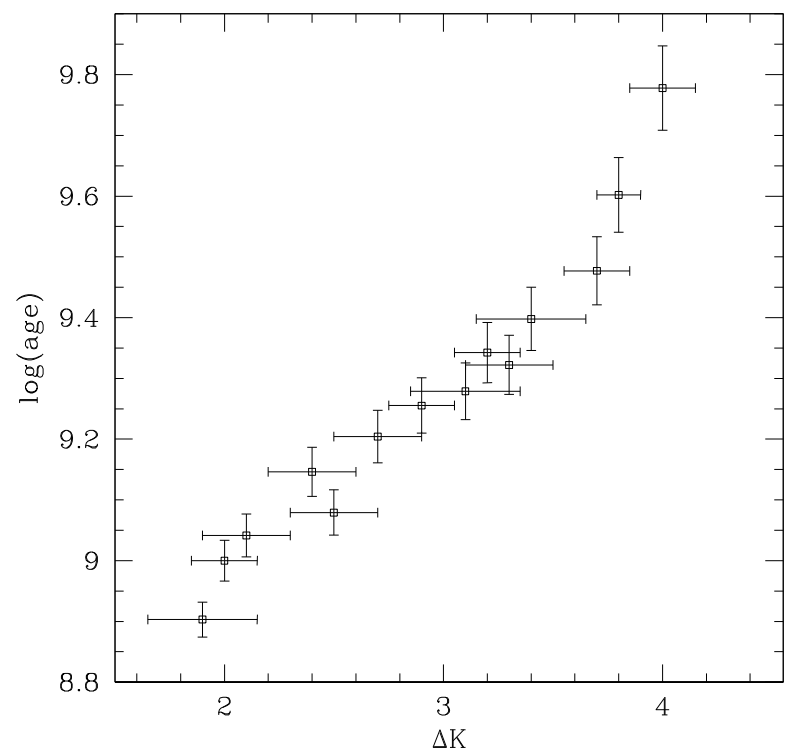

Fig. 2. Age vs. $\Delta K$ relation for the clusters listed in Table 1 . In the same Table we report the uncertainties in age and $\Delta K$.

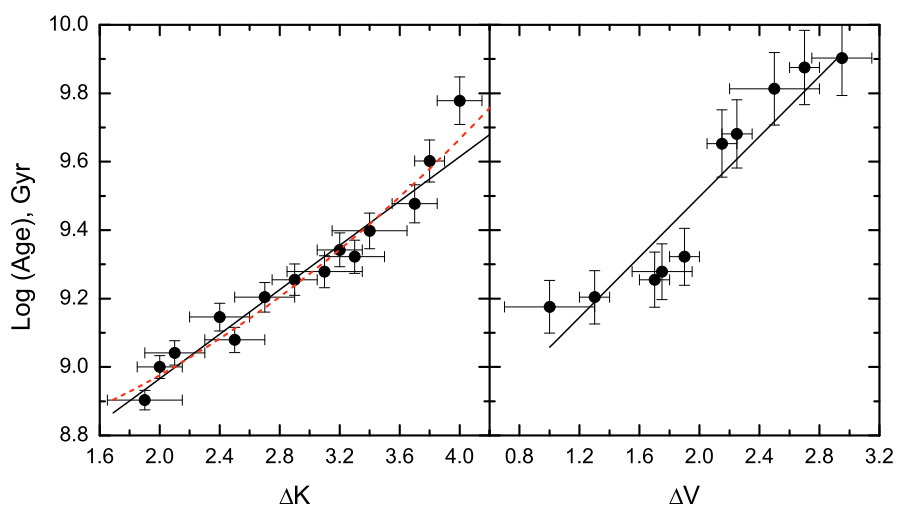

Fig. 3. Left panel: age vs. $\Delta K$ relation for the 14 clusters listed in Table 1. The polynomial fit (Eq. (2)) is showed as a dashed line, and the linear one (Eq. (1)) - as a solid line. Right panel: the optical relation from Carraro \& Chiosi (1994). The solid line is a linear fit through the data.

range. The fits are plotted in the left panel of Fig. 3, while in the right panel we show the optical relation from Carraro \& Chiosi (1994), for the sake of comparison.

As anticipated in the previous Section, the data point distribution in the IR plot (left panel) is smoother than the optical one (right panel), and allow a better fit through the points.

Next, we include the metallicity in the fit, obtaining a 3-dimensional relation:

$$
\begin{aligned}
\log (\text { Age })= & 8.28(0.08)+0.34(0.03) \times \Delta K \\
& +0.00005(0.1) \times[\mathrm{Fe} / \mathrm{H}]
\end{aligned}
$$

with rms $=0.06$ (Fig. 4). The reduced $\chi^{2}$ of the fit is 0.004 .

As expected, the dependence on metallicity is negligible, making $\Delta K$ very useful, since in most cases the newly discovered clusters will not have metallicity estimates available. In comparison with the optical relation (Carraro \& Chiosi 1994, Eq. (2)), the linear relation in the IR is less sensitive to metallicity, and also shows a somewhat shallower dependence on $\Delta K$ (0.34 versus 0.45, see Carraro \& Chiosi 1994).

As for the uncertainty on the age, it is easy to show - by simple propagation - that in the linear cases (Eqs. (2) and (3)),

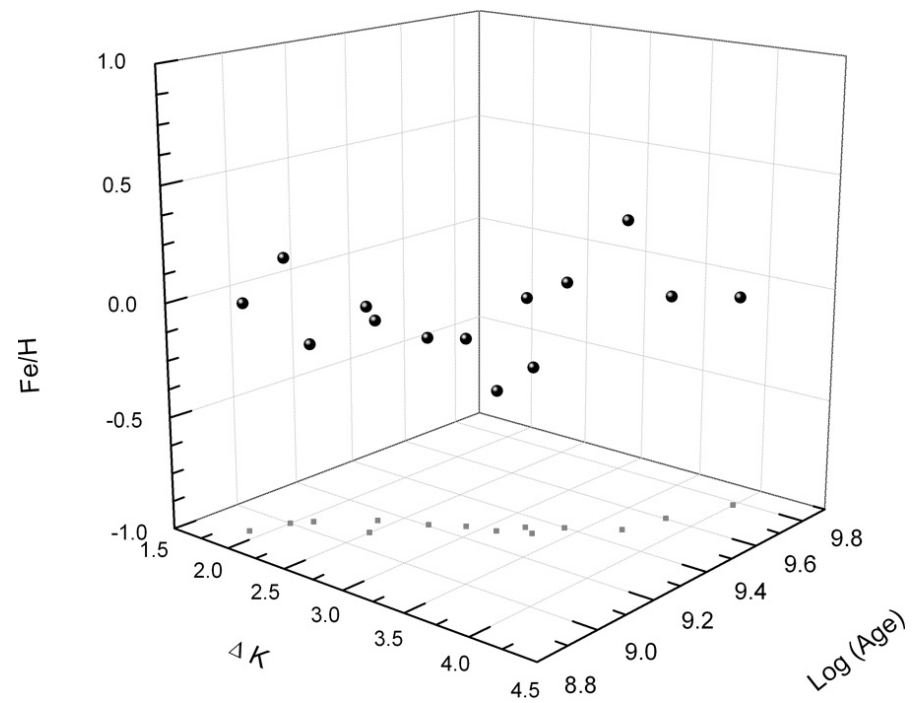

Fig. 4. Age vs. $\Delta K$ for the 14 clusters listed in Table 1, including the metallicity dependence. The projection on the age vs. $\Delta K$ is also shown.

the uncertainty in the age does not depend on the value of $\Delta K$. This is not the case of course for Eq. (2), which has a quadratic dependence on $\Delta K$.

\section{Validation of the new calibrations}

To demonstrate the power of the new calibrations, we applied them to estimate the ages of three clusters with shallow 2MASS photometry, but that have IR photometry from other sources: NGC 6791, NGC 2141, and Berkeley 17. Spectroscopic metallicity estimates are also available for them, allowing us to use all three relations in a metallicity range which encompasses the typical metal content of Galactic open clusters. The results are listed in Table 2. The age uncertainties were derived propagating the measurement errors through the calibrations.

\subsection{NGC 6791}

IR photometry for this cluster is taken from Carney et al. (2005). It was acquired with the IRIM camera at the 4-m Mayall telescope at Kitt Peak National Observatory. The data were photometrically calibrated with the UKIRT standards (Casali \& Hawarden 1992), which were also used to calibrate the 2MASS itself. Not surprisingly, Eq. (37) from Carpenter (2001):

$$
\begin{aligned}
K_{2 \mathrm{MASS}}= & K_{\mathrm{UKIRT}}+0.004(0.006) \times(J-K)_{\mathrm{UKIRT}} \\
& +0.002(0.004)
\end{aligned}
$$

indicates that the color difference $\Delta(J-K) \sim 0.3$ mag between the TO and clump leads to only $0.0012 \pm 0.0018$ mag difference between the $K$-band magnitudes of the two systems - much smaller than our measurement errors. The constant term is negligible for calculating the $\Delta K$ parameters, and it is statistically indistinguishable from zero, anyway.

The CMD of NGC 6791 is shown in the left panel of Fig. 5. Widely accepted values for age and metallicity are in the range of $7-9 \mathrm{Gyr}$ and $[\mathrm{Fe} / \mathrm{H}]=+0.35$ to +0.45 dex, respectively (Carney et al. 2005, and references therein). Our Eqs. (1)-(3), yield ages of 9.3, 6.5 and $6.0 \mathrm{Gyr}$, respectively. 


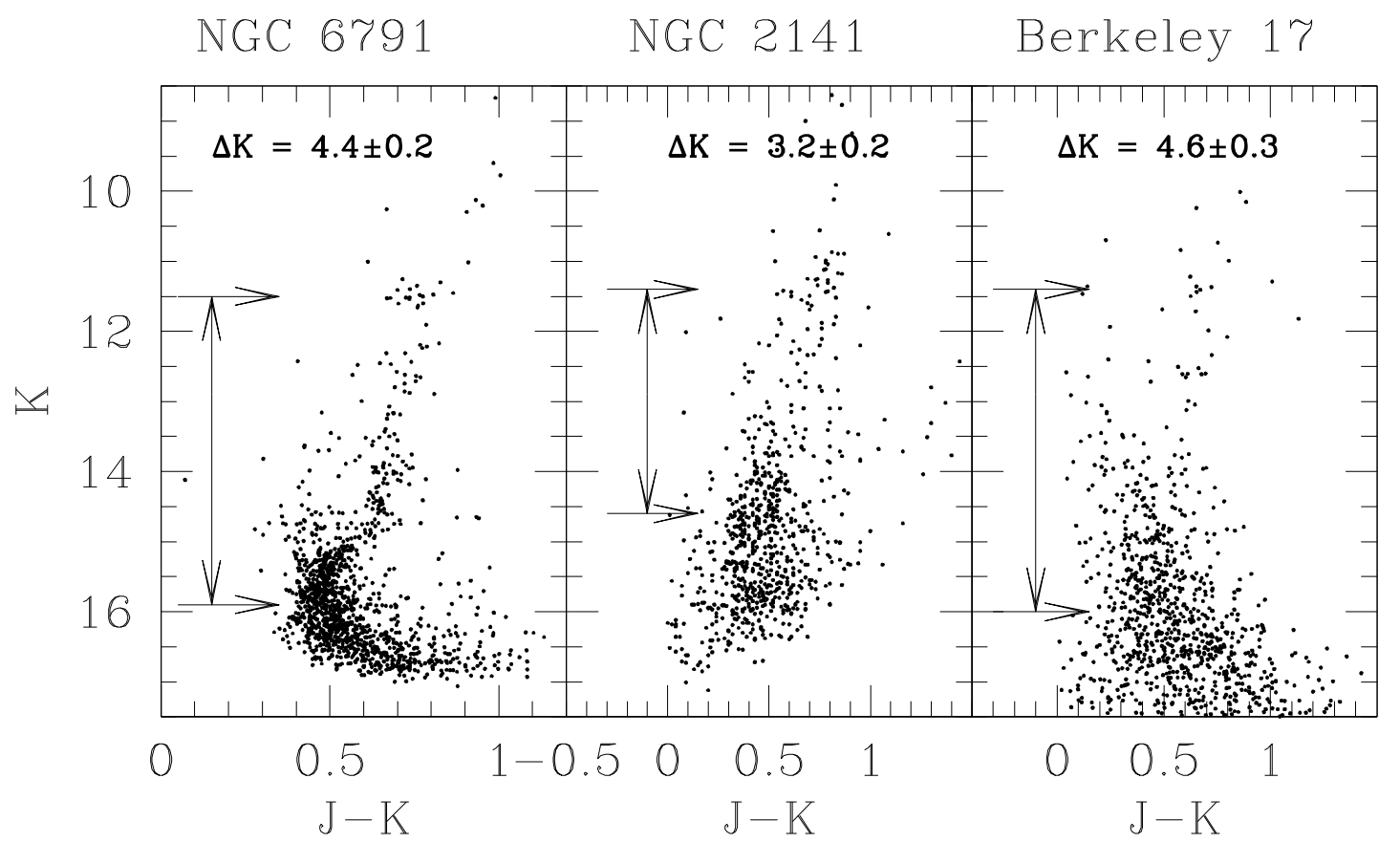

Fig. 5. Application of the new age indicator to NGC 6791, NGC 2141 and Berkeley 17 (from left to right). $\Delta K$ is marked on each CMD.

Table 2. New age estimates for the three test-cases discussed in this paper.

\begin{tabular}{lcccccccc}
\hline \hline \multicolumn{1}{c}{ Name } & $\begin{array}{c}\text { RA } \\
\text { hh:mm:ss }\end{array}$ & $\begin{array}{c}\text { Dec } \\
:^{\prime}:^{\prime \prime}\end{array}$ & $\begin{array}{c}\text { Age } \\
{[\mathrm{Gyr}]}\end{array}$ & {$[\mathrm{Fe} / \mathrm{H}]$} & $\begin{array}{c}\Delta K \\
{[\mathrm{mag}]}\end{array}$ & $\begin{array}{c}\text { Age (1) } \\
{[\mathrm{Gyr}]}\end{array}$ & $\begin{array}{c}\text { Age (2) } \\
{[\mathrm{Gyr}]}\end{array}$ & $\begin{array}{c}\text { Age (3) } \\
{[\mathrm{Gyr}]}\end{array}$ \\
\hline NGC 6791 & $19: 20: 53$ & $+37: 46: 18$ & 8.0 & +0.40 & $4.40 \pm 0.20$ & $9.3 \pm 1.3$ & $6.5 \pm 0.5$ & $6.0 \pm 0.5$ \\
NGC 2141 & $06: 02: 55$ & $+10: 26: 48$ & 2.5 & -0.26 & $3.20 \pm 0.20$ & $2.2 \pm 0.2$ & $2.4 \pm 0.2$ & $2.3 \pm 0.2$ \\
Berkeley 17 & $05: 20: 36$ & $+30: 36: 00$ & 9.0 & -0.10 & $4.60 \pm 0.20$ & $10.0 \pm 1.4$ & $7.6 \pm 0.6$ & $7.0 \pm 0.7$ \\
\hline
\end{tabular}

The last three columns list the ages determined from Eqs. (1)-(3), respectively. References for the literature ages and abundances are given in the text.

\subsection{NGC 2141}

IR photometry for this cluster is taken from Carraro et al. (2001). It was acquired with the ARNICA camera at the Tirgo telescope. The data were photometrically calibrated with the ARNICA standards (Hunt et al. 1998). Equation (8) from Carpenter (2001):

$$
\begin{aligned}
K_{2 \mathrm{MASS}}= & K_{\mathrm{ARNICA}}-0.024(0.011) \times(J-K)_{\mathrm{ARNICA}} \\
& +0.012(0.006)
\end{aligned}
$$

indicates that the color difference $\Delta(J-K) \sim 0.25$ mag between the TO and clump leads to only $\sim 0.006 \pm 0.003$ mag difference between the $K$-band magnitudes of the two systems - again, much smaller than our measurement errors.

The CMD of NGC 2141 is shown in the middle panel of Fig. 5. For this cluster, metallicity is $[\mathrm{Fe} / \mathrm{H}]=-0.26$, and the age is 2.5 Gyr (Carraro et al. 2001). We obtained ages of 2.2, 2.4 and $2.3 \mathrm{Gyr}$, respectively from Eqs. (1)-(3). These results are in good agreement with the isochrone fitting estimates (Carraro et al. 2001).

\subsection{Berkeley 17}

IR photometry for this cluster is taken from Carraro et al. (1999). It was acquired with the ARNICA camera at the Tirgo telescope, and photometrically calibrated with the ARNICA standards (Hunt et al. 1998). As shown in the previous subsection, our age measuring parameter $\Delta K$ is not affected significantly by using a different photometric system than the 2MASS.

The CMD of Berkeley 17 is shown in the right panel of Fig. 5. This cluster is known to have marginally subsolar abundance $[\mathrm{Fe} / \mathrm{H}]=-0.10$ dex and is it $9-10$ Gyr old (Friel et al. 2005). Our calibrations yielded ages of 10.0, 7.6 and 7.0 Gyr, respectively for Eqs. (1)-(3).

This exercise shows that the age of a cluster within the linear regime of our calibrator $(2.5 \leq \Delta K \leq 3.5 \mathrm{mag})$ is closer to the isochrone-derived one when simple linear (Eq. (1)), or the metal dependent (Eq. (3)) fitting are used. The most deviating relation is the polynomial one. In the other two cases of very old clusters, the polynomial relation (Eq. (2)) is the most accurate, and provides age estimates very close to the widely accepted in the literature. Finally, the new calibrations provide, in the linear regime, age estimates that agree quite well with isochrone-based ages, while outside this range the difference might be of the order of $30 \%$.

\section{Summary and conclusions}

In this paper we described relations of the magnitude difference between the TO and the clump $\Delta K$ in the IR CMD versus age for 800-1000 Myr old open star clusters. The age limits are set by the life time of the red clump stars and available photometric data. Our goal is to develop a simple tool to measure the age of newly detected star clusters from the next generation IR sky surveys. The relations are derived in the widely used 2MASS 
photometric system. We provide linear and second order polynomial fits of the age versus $\Delta K$ relationship, and a multi-linear fit which also takes into account the metallicity of the clusters, when available. However, it is unlikely that the metallicity will be immediately available for most of the new objects, so Eqs. (1) and (2) will be mostly used used for en-masse analysis of the new clusters.

Beside, we show that the dependence on metallicity is very shallow, which makes these relations of more robust use than their optical counterpart.

We then illustrate with three examples that the simple linear relation works better for intermediate $\Delta K$ values, while the polynomial relation is preferable at the extremes of the validity range.

The method can potentially be useful for a wider age range, and these calibrations should be extended in the future to cover both younger and older clusters.

Acknowledgements. This research has made use of the SIMBAD database, operated at CDS, Strasbourg, France, and of the WEBDA database maintained by E. Paunzen at Vienna University.

\section{References}

Andreuzzi, G., Bragaglia, A., Tosi, M., \& Marconi, G. 2004, MNRAS, 348, 297 Anthony-Twarog, B. J., \& Twarog, B. A. 1985, ApJ, 291, 595

Anthony-Twarog, B. J., \& Twarog, B. A. 2004, AJ, 127, 1000

Anthony-Twarog, B. J., \& Twarog, B. A. 2006, PASP, 118, 358

Anthony-Twarog, B. J., Tanner, D., Cracraft, M., \& Twarog, B. A. 2006, AJ, 131, 461

Bica, E., Dutra, C. M., Soares, J., \& Barbuy, B. 2003, A\&A, 404, 223

Borissova, J., Pessev, P., Ivanov, V. D., et al. 2003, A\&A, 411, 83
Bruntt, H., Frandsen, S., Kjeldsen, H., \& Andersen, M. I. 1999, A\&AS, 140, 135 Cannon, R. D. 1970, MNRAS, 150, 111

Carney, B., Lee, J.-W., \& Dodson, B. 2005, AJ, 129, 656

Carpenter, J. M. 2001, AJ, 121, 2851

Carraro, G., \& Chiosi, C. 1994, A\&A, 287, 761

Carraro, G., \& Costa, E. 2007, A\&A, 464, 573

Carraro, G., Vallenari, A., Girardi, L., \& Richichi, A. 1999, A\&A, 343, 825

Carraro, G., Hassan, S. M., Ortolani, S., \& Vallenari, A. 2001, A\&A, 372, 879

Casali, M., \& Hawarden, T. 1992, UKIRT/JCMT Newsl., 4, 33

Dias, W. S., Alessi, B. S., Moitinho, A., \& Lépine, J. R. D. 2002, A\&A, 389, 871 Dutra, C. M., \& Bica, E. 2000, A\&A, 359, L9

Dalton, G. B., Caldwell, M., Ward, A. K., et al. 2006, SPIE, 6269, 30

Emerson, J., McPherson, A. M., \& Sutherland, W. 2006, The Messenger, 126, 41

Ferraro, F. R., Messineo, M., Fusi Pecci, F., et al. 1999, AJ, 118, 1738

Friel, E. D., Jacobson, H. R., \& Pilachowski, C. A. 2005, AJ, 129, 2725

Fornal, B., Tucker, D. L., Smith, J. A., et al. 2007, AJ, 133, 1409

Girardi, L., Mermilliod, J.-C., \& Carraro, C. 2000, A\&A, 354, 892

Hunt, L. K., Mannucci, F., Testi, L., et al. 1998, AJ, 115, 2594

Kalirai, J. S., Richer, H. B., Fahlman, G. G., et al. 2001, AJ, 122, 257

Kassis, M., Janes, K. A., Friel, E. D., \& Phelps, R. L. 1997, AJ, 113, 1723

Ivanov, V. D., \& Borissova, J. 2002, A\&A, 390, 937

Ivanov, V. D., Borissova, J., Pessev, P., Ivanov, G. R., \& Kurtev, R. 2002, A\&A, 394, L1

Lawrence, A., Warren, S. J., Alamini, O., et al. 2007, MNRAS, 379, 1599

Lucas, P. W., Hoare, M. G., Longmore, A., et al. 2008, MNRAS, 391, 136

Marconi, G., Hamilton, D., Tosi, M., \& Bragaglia, A. 1997, MNRAS, 291, 763

Meibon, S., Andersen, J., \& Nordstrom, B. 2002, A\&A, 386, 187

Minniti, D., Lucas, P., Adamson, A., et al. 2006, MmSAI, 77, 1184

Phelps, R. L., \& Janes, K. A. 1994, ApJS, 90, 31

Phelps, R. L., Janes, K. A., \& Montgomery, L. A. 1994, AJ, 107, 1079

Rieke, G. H., \& Lebofsky, M. J. 1985, ApJ, 288, 618

Salaris, M., Weiss, A., \& Percival, S. M. 2004, A\&A, 414, 163

Sarajedini, A., Dotter, A., \& Kirkpatrick, A. 2009, ApJ, 698, 1872

Skrutskie, M. F., Cutri, R. M., Stiening, R., et al. 2006, AJ, 131, 1163

Twarog, B. A., Anthony-Twarog, B. J., \& De Lee, N. 2003, AJ, 125, 1383

Vallenari, A., Carraro G., \& Richichi, A. 2000, A\&A, 353, 147 